\title{
The progress of abnormal expression of Lutheran blood group glycoprotein in cancers
}

\author{
Hua Wei, Ying Cui, Yang Chen, Shijie Mu* \\ Department of Blood Transfusion, Tangdu Hospital, the Second Affiliated Hospital of Air Force Military Medical University, Xi'an, \\ Shaanxi 710032, China.
}

\begin{abstract}
Lutheran blood group glycoprotein (Lu-gp) is a specific $\alpha 5$ laminin receptor that is linked by binding to its receptor in the basement membrane matrix. Although the biological function of Lu-gp is unknown, its special affinity with laminin in the chain suggests that it plays an important role in human development and physiological processes. As the interaction between Lu-gp and laminin is further investigated, their expression may be found to play an important role in tumor invasion and metastasis. Laminin receptors help cells adhere, receive and conduct extracellular information into cells, mainly through MAPK pathways, including ERKs, p38MAPK, etc., affecting the degradation of the extracellular matrix and enhancing tumor cell infiltration and metastasis. Present researches in cancers mainly focus on aspects relating to laminin, but largely do not pay attention to the Lutheran blood group antigen, basal cell adhesion molecule. This paper focuses on the abnormal expression of the laminin receptor, that is, the Lutheran blood group antigen, in cancers, which is of great significance to the mechanism of cancer invasion and metastasis, and for finding effective treatment methods for cancers.
\end{abstract}

Keywords: Lutheran blood group, glycoprotein, laminin, cancer

\section{INTRODUCTION}

Cancer is one of the main diseases that greatly endanger human health. The cancer incidence and mortality rate in China account for $23.7 \%$ and $30.0 \%$ of the world, respectively ${ }^{[1-3]}$. This figure is set to further rise due to aging, lifestyle modification, intensification of industrialization and urbanization, etc ${ }^{[4,5]}$. There are more than 5 million patients who die from malignant tumors every year. Angiogenesis, essential for cancer growth, is more frequently found in tumor tissue than normal tissue $^{[6,7]}$ and is known to play a very important role in tumor metastasis, as tumor cells passing through the basement membrane is a key tumor metastasis step. The basement membrane is a mesh consisting of laminin (LN), IV collagen, nidogen, heparan sulfate proteoglycans, and other components, of which $\mathrm{LN}$ is one of the main components. $\mathrm{LN}$ is a family of glycoprotein heterotrimers composed of $\alpha, \beta$ and $\gamma$ chains, with a molecular weight ranging from $80 \sim 100 \mathrm{kDa}$. There are five $\alpha$ chains, four $\beta$ chains, and three $\gamma$ chains known at present ${ }^{[8]}$. Nineteen different LN heterotrimers identified in various cultured cells and tissues are widely expressed in various cell types of both developing and adult tissues, including vascular endothelial and smooth muscle cells ${ }^{[9,10]}$. LN is the most abun-

*Correspondence to: Shijie Mu, Department of Blood Transfusion, Tangdu Hospital, the Second Affiliated Hospital of Air Force Military Medical University, 569 Xinsi Road, Baqiao District, Xi’an, Shaanxi 710032, China. Email: musj1963@fmmu.edu.cn. The authors declared no conflict of interests. 
dant components of the basement membranes surrounding endothelial and medial smooth muscle cells and play a critical role in angiogenesis and the maintenance of vessel architecture through regulation of cell adhesion, proliferation, differentiation, migration, and apoptosis ${ }^{[1]]}$.

\section{LUTHERAN BLOOD GROUP GLYCO- PROTEIN AND LAMININ}

In 1945, Callender et al. found Lutheran blood group glycoprotein (Lu-gp) in the blood of a patient who received repeated blood transfusions. The human body produced Lutheran antibody through immune stimulation such as blood transfusion and pregnancy ${ }^{[12,13]}$.

Lu-gp, also known as basal cell adhesion molecule (BCAM), is a member of the immunoglobulin superfamily (IgSF). Lu-gp binding to intracellular nuclear fibrin serves as a receptor for $\mathrm{LN}$ $\alpha 5$, which has many biological functions, and has important effects, such as mediating cell adhesion and participating in inflammatory and immune responses. The $\mathrm{LN}-\alpha 5$ chain is a constituent of $\mathrm{LN}-$ 511 and LN-521. LN-511 is found in most human basement membranes. Recent studies indicated that Lu-gp mediated cell adhesion to LN-511/LN-521 independently or in concert with integrins ${ }^{[7]}$. As mentioned above, we considered that it implied Lu -gp played an important role in tumor invasion.

Lu-gp, located on red cell membrane, is a member of the IgSF of adhesion molecules, receptors, and signal transducers that bind the extracellular matrix glycoprotein laminin specifically to $\mathrm{LN}$ isoforms containing $\alpha 5$ chains ( $\mathrm{LN}-10 / 11)$. Although the biological roles of Lu-gp are unclear, its specific binding to LN suggests it may play an important role in developmental and physiological processes $^{[8,9]}$.

Currently (and as of 2009) the Lutheran blood group system consists of 19 identified antigens. Among them, 8 are alternate alleles of 4 antithetical antigen pairs, LU1/LU2, LU6/LU9, LU8/LU14, and LU18/LU19, while the other 11 antigens are of very high frequencies. The Lutheran gene is 12.5 $\mathrm{kb}$ and is organized into 15 exons encoding a type I integral membrane including 5 extracellular domains with 597 amino acids. Exon 1 encodes the signal peptide; exons $2 \sim 6$ encode variable segments of the 5 IgSF domains; exons 7 12 encode the 3 constant segments of these domains; exon 13 encodes the transmembrane and cytoplasmic domain; and exons 14 15 encode the C-terminal 40 amino acids of the short transcript of the larger $\mathrm{Lu}$ - gp isoform ${ }^{[10,11]}$.

Lutheran antigens are carried by two kinds of glycoproteins with a relative molecular mass of between $78 \mathrm{kDa}$ and $85 \mathrm{kDa}$ respectively ${ }^{[12]}$. Their structure patterns are shown in Fig. 1. Lu-gp first appears on the surface of nucleated erythrocytes and is then differentiated. Its isomorphism also appears on endothelial cells and epithelial cells of various tissues $^{[13]}$. Recently, data showed that $\mathrm{Lu}-$ $\mathrm{gp}$ expression is increased in some cancers and epithelial cells. Lu-gp is widely distributed in the placenta and organs of adults, such as brain, liver, myocardium, esophagus, skin, cervix, ileum, colon, stomach, and pancreas ${ }^{[10,14]}$.

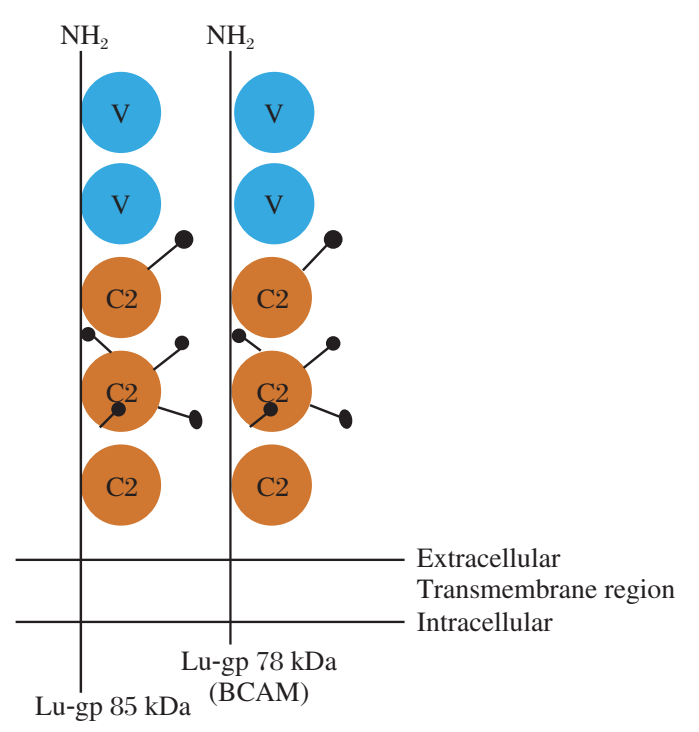

Fig. 1 Structure patterns of $85 \mathrm{kDa}$ and $78 \mathrm{kDa}$ Lu-gp

$\mathrm{Lu}-\mathrm{gp}$ is a specific receptor for $\mathrm{LN}-\alpha 5$. It is widely distributed on the surface of normal epithelial cells, endothelial cells, peripheral nerve cells, macrophages, and most tumor cells. Its binding to $\mathrm{LN}$ in vitro shows high affinity, competitiveness, concentration and time dependence ${ }^{[15]}$. The binding site is the YIGSR sequence of the LN- $\alpha 5$ chain. It has been proved that Lu-gp is a kind of transmembrane glycoprotein which is not only related to tumor metastasis, but also involved in the mutual recognition between cells and the transmission of information inside and outside cells ${ }^{[16]}$. LN receptor on the surface of normal cells is mainly concentrated on the basal side of cells, which causes the cells to adhere to the basement membrane, while $\mathrm{LN}$ receptor on the surface of tumor cells is distributed on the whole cell surface, with an anomalous quantity, most of which cannot be saturated by LN, which provides a molecular basis for the adhesion and invasion of tumor cells. LN receptor 
not only promotes cell adhesion on matrix, but also promotes cell chemotaxis migration and secretion of type IV collagenase ${ }^{[17,18]}$.

A large number of studies have proved that the expression of $85 \mathrm{kDa} \mathrm{LN}$ receptor is closely related to the invasion and metastasis of tumor cells. Tumor cells with high metastatic potential often express more $85 \mathrm{kDa} \mathrm{LN}$ receptors and its precursor ${ }^{[19]}$. The expression of $85 \mathrm{kDa} \mathrm{LN}$ receptor was found related to the stage and metastasis of a significant number of tumors, including lung cancer, colon cancer, ovarian cancer, and cervical cancer. The expression of $85 \mathrm{kDa} \mathrm{LN}$ receptor was also related to the prognosis of breast cancer, which can be used as one of the indicators to determine prognosis. In addition, it was found that synthesized anti-85 kDa LN receptor antibody could significantly inhibit the metastasis of tumor cells ${ }^{[20,21]}$. Satoh et al. also found that inhibiting the expression of $85 \mathrm{kDa} \mathrm{LN}$ receptor can reduce the proliferation of lung cancer cells in vitro ${ }^{[22]}$.

\section{LAMININ}

LN-511 membrane receptors include integrin and non-integrin receptors such as Lu-gp. $\mathrm{LN}-\alpha 5$ is a subunit of LN-511. Many of the biological functions of LN-511 are realized through $\mathrm{LN}-\alpha 5$, because it contains the main site of interaction with the cell receptor. The researches show that $\mathrm{LN}-$ $\alpha 5$ has multiple functions in the development of tissues and organs. Interestingly, $\mathrm{LN}-\alpha 5$ is also a multifunctional adhesion protein which regulates tumor adhesion, invasion, and metastasis ${ }^{[23]}$. On the one hand, $\mathrm{LN}-\alpha 5$ binds to integrin receptor on the surface of tumor cells, which promotes the adhesion of tumor cells; on the other hand, $\mathrm{LN}-\alpha 5$ binds to a class of non-integrin receptor on the surface of tumor cells, which promotes the migration of tumor cells, and is conducive to tumor invasion and metastasis. In fact, there is still a controversial relationship between integrin receptor and non-integrin receptor. Studies have shown that $\mathrm{LN}-\alpha 5$ was highly expressed in breast, thyroid, ovary, prostate, and other tissues ${ }^{[16,18]}$. We found that LN- $\alpha 5$ was also highly expressed in lung cancer, renal cancer, colorectal cancer, pancreatic cancer, and other tumor tissues by immunohistochemistry ${ }^{[15,16,18]}$.

LN- $\alpha 5$ was highly expressed in lung adenocarcinoma tissues, and it was found that the expression of $\mathrm{LN}-\alpha 5$ increased when A549 cells were in non G1 phase ${ }^{[19-21]}$. LN- $\alpha 5$ is also an essential extracellular matrix for neovascularization. Lung adeno- carcinoma cells in the proliferative stage synthesize and secrete $\mathrm{LN}-\alpha 5$, which creates a good environment for angiogenesis of lung adenocarcinoma and promotes neovascularization ${ }^{[23]}$. Angiogenesis promotes the growth of lung adenocarcinoma and forms a vicious circle.

LN-511 is detectable primarily in endothelial cell basement membranes of capillaries and venules commencing $3 \sim 4$ weeks after birth. It is upregulated only by strong proinflammatory signals, such as tumor necrosis factor (TNF- $\alpha$ ), indicating that $\mathrm{LN}-511$ may play an important role in angiogenesis ${ }^{[24]}$. $\mathrm{LN}-511$ receptor on the cell surface are divided into the integrin receptor and non-integrin receptor (such as Lu-gp). The endothelial cell membrane expresses several integrin receptors. Recent studies reported that integrin $\alpha 3 \beta 1$ was the main specific receptor of $L N-511^{[25]}$. Many of the biological functions of LN-511 are mediated through the $\alpha 5$ subunit. The mice lacking $\mathrm{LN}-\alpha 5$ die during late embryogenesis with several developmental defects.

In addition, many studies showed that the expression of $\mathrm{LN}-\alpha 5$ was often maintained or even increased in advanced tumors ${ }^{[21]}$. The results of tumor angiogenesis showed that $\mathrm{LN}-\alpha 5$ was highly expressed in breast cancer and glioma. More literatures proved that $\mathrm{LN}-\alpha 5$ was also highly expressed in lung cancer, renal cancer, colon cancer, pancreatic cancer, and other tumors by immunohistochemistry ${ }^{[25,27]}$.

\section{THE ROLE OF LAMININ AND LAMININ RECEPTOR IN TUMOR METASTASIS}

The interaction between laminin receptor ( $\mathrm{LN}-$ $\mathrm{R}$ ) on the cell surface and $\mathrm{LN}$ in the basement membrane is closely related to the invasion and metastasis of malignant tumor cells. In the process of tumor metastasis, the cancer cells fall from the primary tumor and bind to the matrix complex (such as LN) specifically through their surface receptors ${ }^{[26]}$, then the combined cancer cells secrete or stimulate the host cells to secrete enzymes (such as type IV collagenase) that degrade the matrix locally. In the process, cancer cells must pass through the basement membrane at least three times, breaking through the basement membrane of cancer cells, infiltrating the connective tissue, and passing through the basement membrane of vessels in order to enter and exit the blood vessels ${ }^{[19,20]}$. In recent years, with the wide application of molecular biology techniques and the discovery of other members 
of LN-R family, the study of LN and LN-R has entered a new stage. By observing the effects of $\mathrm{LN}$ on the secretion of type IV collagenase and the infiltration ability in vitro of cholangiocarcinoma cell line QBC939, Wang Shuguang et al. proved that LN can enhance the infiltration ability of cancer cells by stimulating the secretion of type IV collagenase, and that LN antibody can inhibit the stimulating effect of LN on the secretion of type IV collagenase in varying degrees ${ }^{[15]}$.

In a word, as an important part of extracellular matrix, LN has many biological functions by combining with LN-R on the cell surface. One of its important functions is to affect the metastasis behavior of tumor cells. Further studies into the role of $\mathrm{LN}$ and $\mathrm{LN}-\mathrm{R}$ in the process of tumor metastasis will deepen the understanding of the mechanism of tumor metastasis and provide a new way of thinking and treatment for controlling tumor metastasis.

\section{THE RELATIONSHIP BETWEEN LU- THERAN GLYCOPROTEIN AS BASAL CELL ADHESION MOLECULE AND TU- MOR CELL MIGRATION}

BCAM is involved in the interaction between cells and extracellular matrix. It can be roughly divided into five categories: cadherin, selectin, immunoglobulin superfamily, integrin, and hyaluronic acid mucin. BCAM consists of transmembrane glycoproteins, and its molecular structure consists of three parts: extracellular region, transmembrane region, and cytoplasmic region. All of these BCAM regions have extracellular and cytoplasmic functional areas, which are usually activated by intracellular functional areas ${ }^{[15]}$. They are involved in intercellular adhesion, information transmission, inflammation and immune response by binding extracellular junction areas with corresponding ligands.

The interaction between cell matrix is the key for tumor cell migration. Lu-gp is a specific receptor of LN- $\alpha 5$. It competes with integrin to bind to $\mathrm{LN}-\alpha 5$, while $\mathrm{LN}-511$ is the main component of the basement membrane. BCAM interacts with LN10 and $\alpha 5$ of LN-11. On the inner side of the cell, the cytoplasmic tail area interacts with the hemochromatin of the cytoskeleton ${ }^{[9]}$. The interaction between BCAM and hemagglutinin regulates epithelial cell adhesion and proliferation in LN. It has been shown that the expression of Lu-gp can promote the migration of tumor cells in LN-51l rather than cell adhesion. Preferentially binding Lu-gp to $\mathrm{LN}-\alpha 5$ promotes tumor cell migration. $\mathrm{Lu}-\mathrm{gp}$ mainly induces the morphology of spindle cells and pseudopods, and promotes the migration of cells on LN-511. In addition, blocking with Lu-gp antibody/ BCAM antibody can lead to cell flattening, thus inhibiting cell migration on $\mathrm{LN}-511^{[13,14]}$, which is similar to the effect of activating integrin 1 antibody. Researchers believe that Lu-gp is required to competitively regulate cell adhesion through integrin for tumor cell migration on LN-511, and Lu$\mathrm{gp}$ is also required to competitively weaken cell adhesion through integrin for tumor cell migration on LN-511. We believe that this competitive interaction participates in the balance between cell stasis and migration, and the competition between LN receptors may also provide a balance between cell stasis and migration behaviors.

\section{EXPRESSION OF LUTHERAN GLYCO- PROTEIN IN TUMOR CELLS}

It was reported that Lu-gp expression was enhanced in the process of arterial development, erythropoiesis, smooth muscle development and other organ formation, certain cancers, and epithelial cell malignant transformation ${ }^{[11,12]}$. In addition, the adhesion of red blood cells to endothelial cells is related to vascular complications in diabetic patients, leading to endothelial disorders or vascular dysfunction, such as organ transplant rejection, systemic lupus erythematosus and a series of vasculitis and thrombosis disorders. So it may play an important role in cancers.

Lu-gp has also been reported to be highly expressed not only in benign and malignant skin tumors (squamous and basal cell carcinoma, keratomyoma and verruca), but also in normal skin, psoriasis and contact dermatitis ${ }^{[10,13-14]}$. In addition, Lu-gp was found to increase significantly in patients' sera in a study by mass spectrometry to analyze serum biomarkers of pancreatic cancer ${ }^{[15]}$. Lu-gp was also highly expressed in endometrial carcinoma and ovarian endometrial carcinoma by immunohistochemistry ${ }^{[16]}$. Similarly, Lu-gp was also highly expressed in liver cancer, and in surrounding tissues and nodules ${ }^{[17]}$. More importantly, the concentration of Lu-gp in serum of patients with liver cancer decreased significantly with the removal of liver cancer tissue ${ }^{[18]}$. However, Lu-gp expression is not only related to malignant tissues ${ }^{[10]}$, it is also expressed in benign epithelial skin tumors and normal tissues near liver cancer ${ }^{[17]}$. Lu-gp is only expressed in primary tumor cells, but not in metastatic tumor cells ${ }^{[19]}$.

However, the expression of Lu-gp in papillary 
thyroid cancer with lymph node metastasis and papillary thyroid cancer with lymphocytic infiltration was lower than that in papillary thyroid cancer without the above characteristics, and the data in both groups were lower than that in the control group with normal thyroid tissue. The expression of Lu-gp in papillary thyroid carcinoma with diameter of $>10 \mathrm{~mm}$ was significantly lower than that in papillary thyroid carcinoma with diameter of $<10 \mathrm{~mm}$ while the data in both groups were lower than that in the normal thyroid tissue control group ${ }^{[9]}$.

In contrast, BCAM expression was lower in thyroid cancer tissue samples, whereas high Lu-gp expression was also detected in human renal pelvis, ureteral, and bladder tumors, significantly associated with advanced tumors $(P=0.02)$. Patients with high Lu-gp expression showed a tendency for increased tumor volume and decreased diseasespecific survival. More importantly, reports have found that H-Rasv 12 mutants can transcriptionally upregulate Lu/BCAM expression as showed in Fig. 2. The study suggests that cells activate BCAM overexpression through ligand layer adhesion protein 10/11, further enhance colony formation and anchor independence cell migration, RhoA elevation, and inhibition of Racl activity through cell growth, adhesion, and suppress cell migration through the Erk signaling pathway. RhoA is involved in actin stress fiber formation and focal adhesion $^{[27]}$.

That is, in the presence of LN, Lu-gp can promote the rearrangement of $\mathrm{F}$-actin, increase the adhesion of tumor cells and reduce cell migration through the Erk and RhoA/Racl signaling pathway, resulting in increased cell adhesion, promotion of F-actin lesion formation and tumor formation. A hypothetical model of the Lu-gp mediated cell adhesion signaling pathway during LN stimulation of human bladder tumorigenesis is shown in Fig. $\mathbf{2}^{[27]}$. Studies Show the importance of Lu-gp in cancer progression, but its potential mechanisms in lung cancer are less studied.

In a comprehensive experimental study on tissue sections, researchers found that an antibody could inhibit the binding of Lu-gp to LN- $\alpha 5$. In order to recognize the blocking antibody, the site of Lu-gp through this antibody was identified. The extracellular domain of Lu-gp contains five IgSF regions, D1-D2-D3-D4-D5. The antibody epitope was located in D2, not in D3, the main binding site of LN- $\alpha 5^{[28,29]}$. In addition, mutation studies show that $\arg 175$ (i.e. LU4 blood group antigen site) is essential for the formation of antigen epitopes,

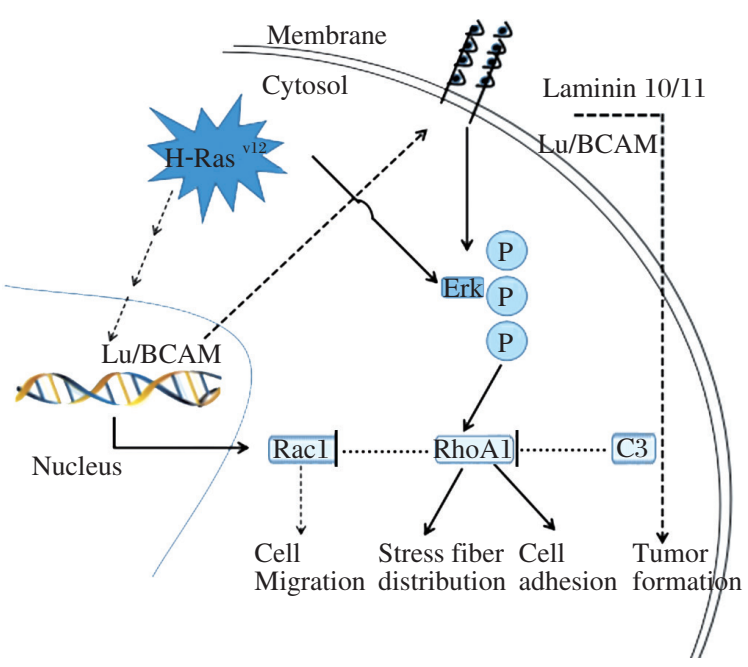

Fig. 2 Hypothesis model of Lu-gp mediated cell adhesion signaling pathway in the process of bladder tumor ${ }^{[27]}$

and the antibody is tightly bound in space, which hinders its interaction with $\alpha 5$. Moreover, the cell adhesion test using this antibody also showed that Lu-gp, as an auxiliary receptor, could make tumor cells adhere to $\mathrm{LN}-\alpha 5^{[30]}$. The conclusion of this study showed that this inhibitory antibody, a functional blocking antibody against Lu/BCAM, can not only be used to study cell adhesion to $\mathrm{LN}-\alpha 5$, but can also be used to develop drug to inhibit vascular obstruction of sickle cells ${ }^{[22,31]}$.

As an important part of the extracellular matrix, $\mathrm{LN}$ combines with the $\mathrm{LN}$ receptor on the cell surface to produce a variety of biological functions, one of which is to affect the invasion and metastasis of tumor cells. Lu-gp is a highly specific membrane receptor protein of $\mathrm{LN}-\alpha 5$ and further study on its role and $\mathrm{LN}$ in the process of liver tumor metastasis will deepen the understanding of the mechanism of liver tumor metastasis and provide a new way of treatment for the control of cancer invasion and metastasis.

\section{References}

[1] Zeng H, Chen W, Zheng R, et al. Changing cancer survival in China during 2003-15: a pooled analysis of 17 population-based cancer registries[J]. Lancet Glob Health, 2018, 6(5): 555-67.

[2] Chen W, Sun K, Zheng R, et al. Cancer incidence and mortality in China, 2014[J]. Chin J Cancer Res, 2018, 30(1): 1-12.

[3] Zhou B, Zong S, Zhong W, et al. Interaction between laminin-5 $\gamma 2$ and integrin $\beta 1$ promotes the tumor budding of colorectal cancer via the activation of Yesassociated proteins[J]. Oncogene, 2019, 39(5): 152742.

[4] Chen W, Zheng R, Baade PD, et al. Cancer statistics 
in China, 2015[J]. CA Cancer J Clin, 2016, 66(2): $115-32$.

[5] Torre LA, Siegel RL, Jemal A. Lung cancer statistics[J]. Adv Exp Med Biol, 2015, 893(12): 1-19.

[6] Islami F, Chen W, Yu XQ, et al. Cancer deaths and cases attributable to lifestyle factors and infections in China, 2013[J]. Ann Oncol, 2017, 28(10): 2567-74.

[7] Willberg J, Hormia M, Takkunen M, et al. Lutheran blood group antigen as a receptor for $\alpha 5$ laminins in gingival epithelia[J]. J Periodontol, 2007, 78(9): 1810-8.

[8] Manders DB, Kishore HA, Gazdar AF, et al. Dysregulation of fibulin-5 and matrix metalloproteases in epithelial ovarian cancer[J]. Oncotarget, 2018, 9(18): 14251-67.

[9] Langer IBV, Visentainer JEL, Zacarias JMV, et al. Genotyping of Dombrock and Lutheran blood group systems in blood donors from the southwestern region of the state of Paraná, Southern Brazil[J]. Hematol Transfus Cell Ther, 2019, 41(1): 25-30.

[10] Kikkawa Y, Miner JH. Review: Lutheran/b-CAM: A lamin receptor on red blood cells and in various tissues[J]. Connect Tissue Res, 2005, 46(6): 193-9.

[11] Gordon-Weeks A, Lim SY, Yuzhalin A, et al. Tumour-derived laminin $\alpha 5$ (LAMA5) promotes colorectal liver metastasis growth, branching angiogenesis and notch pathway inhibition[J]. Cancers, 2019, 11(5): 630.

[12] Crew VK, Poole J, Banks J, et al. LU21: a new highfrequency antigen in the Lutheran blood group system[J]. Vox Sanguin, 2004, 87(9): 109-13.

[13] Lin Y, Ge X, Zhang X, et al. Protocadherin-8 promotes invasion and metastasis via laminin subunit $\gamma 2$ in gastric cancer[J]. Cancer Sci, 2018, 109(3): 732-40.

[14] Daniels G. An update on the Lutheran blood group system[J]. Immunohematology, 2019, 35(1): 23-4.

[15] Wang SG, Han BL, Chen YS, et al. Significance of laminin in tumor cell invasion in cholangiocarcinoma[J]. Acta Acdemiae Militaris Tertiae (in Chinese), 1995, 17(5): 389-92.

[16] Brocks DG, Strecker H, Neubauer HP, et al. Radioimmunoassay of laminin in serum and its application to cancer patients[J]. Clin Chem, 1986, 32(5): 787-91.

[17] Parsons SF, Mallinson G, Holmes CH, et al. The Lutheran blood group glycoprotein, another member of the immunoglobulin superfamily is widely expressed in human tissues and is developmentally regulated in human liver[J]. Proc Natl Acad Sci USA, 2016, 92(12): 5496-500.

[18] Latini FR, Bastos AU, Arnoni CP, et al. DARC and BCAM (Lutheran) reduced expression in thyroid cancer[J]. Blood Cells Mol Dis, 2013, 50(2): 161-5.

[19] Kikkawa Y, Ogawa T, Sudo R, et al. The lutheran/basal cell adhesion molecule promotes tumor cell migration by modulating integrin-mediated cell attachment to laminin-511 protein[J]. J Biol Chem, 2013, 288(43): 30990-1001.

[20] Murphy MM, Zayed MA, Evans A, et al. Role of Rap 1 in promoting sickle red blood cell adhesion to laminin via BCAM / LU[J]. Blood, 2005, 105(8): 3322-9.

[21] Drewniok C, Wienrich BG, Schon M, et al. Molecular interactions of B-CAM (basal-cell adhesion molecule) and laminin in epithelial skin cancer[J]. Arch Dermatol Res, 2004, 296(2): 59-66.

[22] Satoh K, Narumi K, Abe T, et al. Diminution of 37$\mathrm{kDa}$ laminin binding protein expression reduces tumour formation of murine lung cancer cells[J]. $\mathrm{Br} J$ Cancer, 1999, 80(8): 1115-22.

[23] Bernemann TM, Podda M, Wolter M, et al. Expression of the basal cell adhesion molecule (B-CAM) in normal and diseased human skin[J]. J Cutan Pathol, 2000, 27(3): 108-11.

[24] Drewniok C, Wienrich BG, Schön M, et al. Molecular interactions of B-CAM (basal-cell adhesionmolecule) and laminin in epithelial skin cancer[J]. Dermatol Res, 2004, 296(2): 59-66.

[25] Schön M, Klein CE, Hogenkamp V, et al. Basal-cell adhesion molecule (B-CAM) is induced in epithelial skin tumors and inflammatory epidermis, and is expressed at cell-cell and cell-substrate contact sites[J]. J Invest Dermatol, 2000, 115(6): 1047-53.

[26] Manders DB, Kishore HA, Gazdar AF, et al. Dysregulation of fibulin-5 and matrix metalloproteases in epithelial ovarian cancer[J]. Oncotarget, 2018, 9 (18): 14251-67.

[27] Chang HY, Chang HM, Wu TJ, et al. The role of Lutheran/basal cell adhesion molecule in human bladder carcinogenesis[J]. J Biomed Sci, 2017, 24(1): 61.

[28] Caires-Dos-Santos L, da Silva SV, Smuczek B, et al. Laminin derived peptide $\mathrm{C} 16$ regulates Tks expression and reactive oxygen species generation in human prostate cancer cells[J]. J Cell Physiol, 2020, 235(1): 587-98.

[29] Yu KH, Barry CG, Austin D, et al., Stable isotope dilution multidimensional liquid chromatography-tandem mass spectrometry for pancreatic cancer serum biomarker discovery[J]. J Proteome Res, 2009 (8): 1565-76.

[30] Kikkawa Y, Miwa T, Tanimizu N, et al. Soluble Lutheran/basal cell adhesion molecule is detectable in plasma of hepatocellular carcinoma patients and modulates cellular interaction with laminin-511 in vitro[J]. Exp Cell Res, 2014, 328(2): 197-206.

[31] Yellapurkar S, Natarajan S, Boaz K, et al. Expression of Laminin in oral squamous cell carcinomas[J]. Asian Pac J Cancer Prev, 2018, 19(2): 407-13.

Received 20 March 2020, Revised 11 May 2020, Accepted 04 June 2020 